\title{
PAHs in the halo of NGC 5529
}

\author{
J. A. Irwin ${ }^{1}$, H. Kennedy ${ }^{1}$, T. Parkin ${ }^{1}$, and S. Madden ${ }^{2}$ \\ 1 Dept. of Physics, Engineering Physics, \& Astronomy, Queen's University, Kingston, K7L 3N6, Canada \\ e-mail: irwin@astro.queensu.ca \\ 2 CEA/Saclay, Service d'Astrophysique, Orme des Merisiers, Bâtiment 709, 91191 Gif-sur-Yvette Cedex, France \\ e-mail: smadden@cea.fr
}

Received 26 April 2007 / Accepted 28 August 2007

\section{ABSTRACT}

\begin{abstract}
We present sensitive ISO $\lambda 6.7 \mu \mathrm{m}$ observations of the edge-on galaxy, NGC 5529, finding an extensive MIR halo around NGC 5529 . The emission is dominated by PAHs in this band. The PAH halo has an exponential scale height of $3.7 \mathrm{kpc}$ but can still be detected as far as $\approx 10 \mathrm{kpc}$ from the plane to the limits of the high dynamic range (1770/1) data. This is the most extensive PAH halo yet detected in a normal galaxy. This halo shows substructure and the PAHs likely originate from some type of disk outflow. PAHs are long-lived in a halo environment and therefore continuous replenishment from the disk is not required (unless halo PAHs are also being destroyed or removed), consistent with the current low SFR of the galaxy. The PAHs correlate spatially with halo H $\alpha$ emission, previously observed by Miller \& Veilleux (2003, ApJS, 148, 383); both components are likely excited/ionized by in-disk photons that are leaking into the halo. The presence of halo gas may be related to the environment of NGC 5529 which contains at least 17 galaxies in a small group of which NGC 5529 is the dominant member. Of these, we have identified two new companions from the SDSS.
\end{abstract}

Key words. galaxies: general - galaxies: individual: NGC 5529 - galaxies: halos - galaxies: ISM

\section{Introduction}

Nearby edge-on galaxies provide an important local laboratory for understanding physical conditions in the disk-halo interface. This critical region separates two very different environments over a relatively small vertical distance. It plays an important role in a galaxy's energy balance as well as its chemical evolution, since outflows from the disk, circulating "fountains", and possibly infalling external material may each be involved at some point in a galaxy's evolution. Moreover, knowledge of the physical conditions of outflowing gas in nearby galaxies can provide important constraints on galaxy formation scenarios, since outflows (i.e. some form of "feedback") are crucial to these models (e.g. Marri \& White 2003, and others). Nearby edgeon galaxies have now been studied in many wavebands showing a variety of interstellar medium (ISM) components above the galactic disk. Indeed, in at least one galaxy that has a high star formation rate (SFR), every component of the interstellar medium, including dust, molecular gas, HI, extra-planar diffuse ionized gas (eDIG, detected by $\mathrm{H} \alpha$ emission) and X-ray emitting hot gas has been detected above the galactic disk (see Lee et al. 2001; and Brar et al. 2003). In each component, discrete disk-halo vertical structures are seen, suggestive of outflow.

The recent discovery, from mid-infrared (MIR) observations, of PAH (polycyclic aromatic hydrocarbon) emission ${ }^{1}$ in the halo of the low SFR galaxy, NGC 5907 (Irwin \& Madden 2006), has prompted a search for other galaxies that may show similar emission. In this paper, we present results for the edge-on galaxy, NGC 5529, using archival data from the Infrared Space

1 There is some uncertainty as to the nature of the carriers of the MIR band emission. In this paper, we adopt the PAH nomenclature for consistency with other authors (e.g. Draine \& Li 2007).
Observatory (ISO) in the $\lambda 6.75 \mu \mathrm{m}$ waveband, which selects the $\lambda 6.2, \lambda 7.7$, and part of the $\lambda 8.6 \mu \mathrm{m}$ PAH features (see Fig. 7 of Irwin \& Madden 2006). These were the only ISO observations taken of this galaxy. In Sect. 2, we introduce the galaxy, Sect. 3 presents the observations and data reduction, Sect. 4 lists the results, and the discussion and conclusion are presented in Sects. 5 and 6 , respectively.

\section{NGC 5529 and its environment}

NGC 5529, whose basic parameters are listed in Table 1, is an edge-on galaxy with a prominent dust lane. It is classified as an Sc galaxy, but is likely barred (Kregel \& van der Kruit 2004) and a peanut-shaped bulge is also apparent in the optical image shown in Fig. 1. At a distance of $D=43.9 \mathrm{Mpc}$ ( 1 arcsec $=213 \mathrm{pc}$ ), this galaxy is physically very large (diameter, $D=81 \mathrm{kpc}$ ). However, its properties related to star formation (SF) are similar to those of NGC 5907 (see Table 1) which is half its size. The SFRs of both galaxies are modest, i.e. 1 to $3 M_{\odot} \mathrm{yr}^{-1}$; by comparison, the SFR of the better-known quiescent galaxy, NGC 891, is $3.8 M_{\odot} \mathrm{yr}^{-1}$ (Popescu et al. 2000). With an inclination of $i=90^{\circ}$ and an SFR that is similar to a galaxy in which PAH halo emission has already been detected, NGC 5529 is an ideal candidate for the study of extraplanar emission in general, and the search for high latitude PAHs in particular.

To our knowledge, only one recent search for extraplanar emission in NGC 5529 has been carried out. This involved an $\mathrm{H} \alpha / \mathrm{N}[\mathrm{II}]$ observation (hereafter referred to as $\mathrm{H} \alpha$ ) by Miller $\&$ Veilleux (2003), with a positive detection. These authors report widespread eDIG within the central $10 \mathrm{kpc}$ of the disk and find a weak, extended component with an average exponential scale height of $z_{\mathrm{e}}=4.5 \mathrm{kpc}$. The total eDIG mass, $M_{\mathrm{eDIG}}$, was 


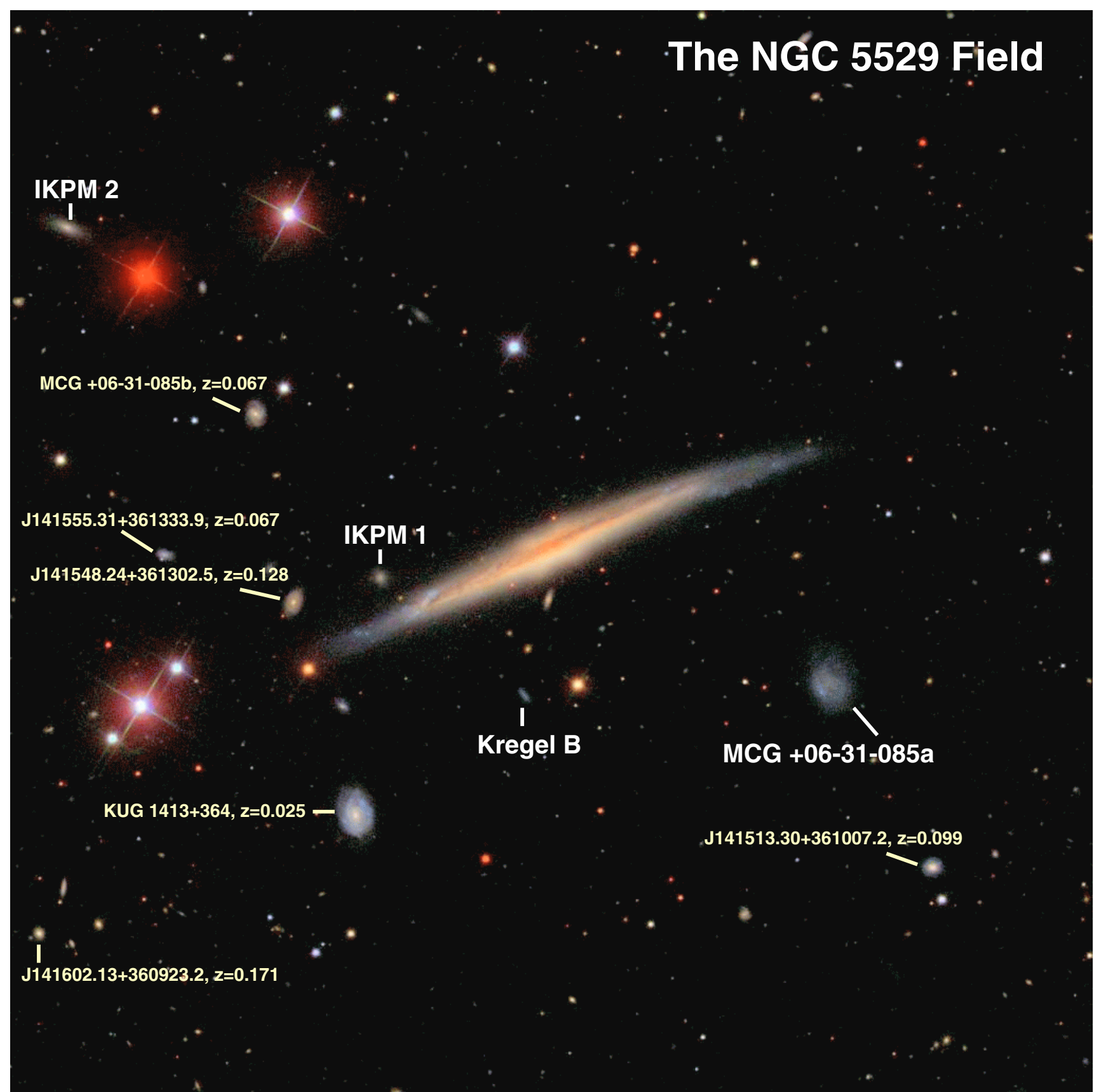

Fig. 1. Optical image of NGC 5529 and its near environment, from SDSS DR5. The displayed field of view is $11.95^{\prime}$ on a side. Companions to NGC 5529 are marked in white (see Table 2) and background galaxies in this field for which spectroscopic redshifts are known are marked in yellow. The spectroscopic redshift of NGC 5529 is $z=0.00959$.

found to be $M_{\mathrm{eDIG}}=2.0 \times 10^{8} M_{\odot}$. By contrast, an early radio continuum observation did not detect extraplanar radio emission, but the observations were likely not sensitive enough to do so (Hummel et al. 1991). In the soft X-ray band, an upper limit of $L_{\mathrm{X}}=2.2 \times 10^{41} \mathrm{erg} \mathrm{s}^{-1}$ (scaled to our adopted distance, and so throughout) has been placed on the galaxy's total X-ray emission (Benson et al. 2000). The stellar component of NGC 5529 has been widely studied, however, and various authors have reported a thick stellar disk, although optical measurements have been hindered, to some extent, by the prominent dust lane (Fig. 1). For example, vertical fits to $B, V$, and $I$-band data give $z_{\mathrm{s}}=0.99,0.92$, and $0.86 \mathrm{kpc}$, respectively, where the best fit is to a $\operatorname{sech}\left(|z| / z_{\mathrm{s}}\right)$ function (de Grijs \& van der Kruit 1996). More recently, exponential fits to $R$-band data result in a mean value of $z_{\mathrm{e}}=8^{\prime \prime}(1.7 \mathrm{kpc})$, increasing with galactocentric distance (Schwarzkopf \& Dettmar 2001), an effect that may be related to the fact that this galaxy is undergoing an interaction (see below). The Two Micron All Sky Survey (2MASS) $K_{\mathrm{s}}$-band scale height is $z_{\mathrm{s} 2}=0.98 \mathrm{kpc}$ (scaled to our distance) where, in this case, the luminosity volume is fit by a $\operatorname{sech}^{2}\left(|z| / z_{\mathrm{s} 2}\right)$ function (Bizyaev \& Mitronova 2002).

In a series of recent papers by Kregel et al. (Kregel et al. 2004a,b; Kregel \& van der Kruit 2004, 2005; and Kregel et al. 2005), which include optical long-slit spectroscopy and HI observations (HI data are also available from Rhee \& van Albada 1996), the authors have looked in detail at the structure and kinematics of edge-on disks, including NGC 5529. Among their findings are the following. The NW side of the galaxy is advancing 
Table 1. Galaxy parameters.

\begin{tabular}{lcc}
\hline \hline Parameter & NGC 5529 $^{a}$ & NGC 5907 $^{b}$ \\
\hline RA $(\mathrm{J} 2000)(\mathrm{h} \mathrm{m} \mathrm{s})$ & 141534.07 & 151553.69 \\
Dec $(\mathrm{J} 2000)\left({ }^{\circ}{ }^{\prime \prime}\right)$ & 361335.7 & 561943.9 \\
$V_{\mathrm{hel}}{ }^{c}\left(\mathrm{~km} \mathrm{~s}^{-1}\right)$ & 2875 & 667 \\
Distance $(\mathrm{Mpc})$ & $43.9^{d}$ & 11 \\
Morphological type & $\mathrm{Sc}$ & $\mathrm{SA}(\mathrm{s}) \mathrm{c}$ : sp HII: \\
$2 a \times 2 b^{e}\left({ }^{\prime} \times \times^{\prime}\right)$ & $6.35 \times 0.64$ & $12.77 \times 1.40$ \\
$D^{f}(\mathrm{kpc})$ & 81 & 41 \\
Inclination $\left.^{\circ}\right)$ & $90^{g}$ & 86.5 \\
$f_{12}, f_{25}, f_{60}, f_{100}{ }^{h}(\mathrm{Jy})$ & $0.255,0.234,1.95,7.73$ & $1.29,1.44,9.14,37.43^{i}$ \\
$f_{60} / f_{100}$ & 0.25 & 0.24 \\
$L_{\mathrm{FIR}}, L_{\mathrm{IR}}{ }^{j}\left(L_{\odot}\right)$ & $9.7 \times 10^{9}, 1.9 \times 10^{10}$ & $2.9 \times 10^{9}, 5.8 \times 10^{9} k$ \\
$L_{\mathrm{FIR}} / A^{l}\left(\mathrm{erg} \mathrm{s}^{-1} \mathrm{kpc}^{-2}\right)$ & $7.2 \times 10^{39}$ & $8.4 \times 10^{39}$ \\
$S F R^{m}\left(M_{\odot} \mathrm{yr}^{-1}\right)$ & 3.3 & $1.0^{n}$ \\
$S F R / \mathrm{A}^{o}\left(M_{\odot} \mathrm{yr}^{-1} \mathrm{kpc}^{-2}\right)$ & $6.5 \times 10^{-4}$ & $7.6 \times 10^{-4}$ \\
\hline
\end{tabular}

${ }^{a}$ Values from the NASA Extragalactic Database (NED) unless otherwise indicated. ${ }^{b}$ Values from Irwin \& Madden (2006), or calculated from this table, unless otherwise indicated. ${ }^{c}$ Heliocentric radial velocity. ${ }^{d}$ From Tully (1988). The distance to the group, GH 141 , is $D=49.1 \mathrm{Mpc}$ (van Driel et al. 2001). We assume $H_{0}=75 \mathrm{~km} \mathrm{~s}^{-1} \mathrm{Mpc}^{-1}$ throughout. ${ }^{e}$ Major $\times$ minor axis angular size. ${ }^{f}$ Major axis linear size. ${ }^{g}$ From Tully (1988). ${ }^{h}$ Infrared fluxes at $\lambda 12,25,60$ and $100 \mu \mathrm{m}$, respectively. ${ }^{i}$ From Sanders et al. (2003). ${ }^{j}$ Far infrared (FIR) and infrared (IR) luminosity, respectively, using the formalism of Sanders \& Mirabel (1996) with their constant, $C=1$, and $L_{\odot}=3.84 \times 10^{33} \mathrm{erg} \mathrm{s}^{-1}$. $^{k}$ Note that the FIR luminosity differs slightly from the value of Irwin \& Madden (2006) since the latter authors use a different formalism than described in note $j$. ${ }^{l}$ FIR luminosity per unit area, where $A=\pi D^{2} / 4$. See also note $k .{ }^{m}$ Star formation rate, using $L_{\mathrm{IR}}$ and the formalism of Kennicutt (1998). ${ }^{n}$ This value could be as high as $S F R=2.2 M_{\odot} \mathrm{yr}^{-1}$ (Misiriotis et al. 2001). ${ }^{o}$ See note $l$.

and the SE is receding with respect to its center. The optical distribution is asymmetric, being more extended on the NW than the SE side (not obvious in Fig. 1 but see Kregel et al. 2004a). The HI distribution shows the classical double-horned profile of a rotating disk galaxy, but non-circular motions are seen in the inner parts. Of particular interest is their discovery of HI bridges that connect two of the galaxy's companions, MCG+06-31-085a and Kregel B (both labelled in Fig. 1), to the disk of NGC 5529. Thus, the galaxy is clearly interacting. A high velocity dispersion in the disk is further evidence that the disk is perturbed.

The richness of the field within which NGC 5529 is located may have some bearing on the activity in NGC 5529 and its halo emission (see Sect. 4.3). Therefore, we have listed the group members and known associated galaxies, from literature sources, in Table 2. The total number of previously known group members is 15 galaxies, of which NGC 5529 is dominant. In addition, since NGC 5529 can be found in the Sloan Digital Sky Survey 5th data release (SDSS DR5, Adelman-McCarthy et al. 2007), we have searched this data set for additional group members within a square region of angular size, $27^{\prime}(345 \mathrm{kpc})$ on a side and within a velocity range of $\pm 500 \mathrm{~km} \mathrm{~s}^{-1}$ with respect to NGC 5529. There are a total of 301 non-stellar objects in this field (including NGC 5529) of which 35 have spectroscopic redshifts $\left(z_{\mathrm{sp}}\right)$ and all have photometric redshifts $\left(z_{\mathrm{ph}}\right)$. Although some of the objects which have only photometric redshifts could be companions, we have discounted these from our search because $z_{\text {ph }}$ values are unreliable. For example, the $z_{\text {ph }}$ values for NGC 5529 and Kregel B imply incorrect velocities (see values in parentheses in Table 2$)^{2}$. Two new companions have been found and are listed as IKPM 1 and IKPM 2 (along with their SDSS identifiers) in Table 2. The newly identified companions are also labelled in Fig. 1.

${ }^{2}$ A plot of $z_{\text {ph }}$ against $z_{\mathrm{sp}}$ for the 35 galaxies in the field for which both values exist, show typical discrepancies of $\Delta z \approx 0.07$ which is in good agreement with that found by Fernández-Soto et al. (2001) for low redshift objects in the Hubble Deep Field.

\section{Observations and data reduction}

The ISO $\lambda 6.75 \mu \mathrm{m}$ observations were taken on three different days (see Table 3) using the infrared camera, ISOCAM, and each data set had a different on-source pointing position. The observations were taken in beam-switching mode which involves obtaining a sequence of frames on-source, each of $10 \mathrm{~s}$ integration time, followed by a sequence of off-source sky pointings at various positions around the galaxy. There were 26 different offsource positions, which are listed in Table 4. Each data set contained a total of 675 frames, of which approximately $40 \%$ were on-source pointings. The off-source sky frames were used for the flat field, its distortions, and the mean sky value at each pixel. This mode of operation provides an accurate means of making sky and instrumental corrections that are appropriate for the observing conditions, rather than relying on a flat-field library, resulting in high dynamic-range results. Each data set was then reduced separately, as described below.

All data were reduced using the CAM Interactive Reduction Package (CIR, Chanial 2003). First, the dark current was subtracted following the method of Biviano et al. (1998) which includes a dark correction, a second-order dark correction depending on detector temperature and time of observation, and a shortdrift correction. Next, high glitches due to cosmic ray (CR) hits were removed automatically via a multi-resolution filtering technique (Starck et al. 1999) at a $6 \sigma$ level. The effects of memory on a pixel (transient effects) were then corrected (de-glitched) using the Fouks-Schubert method (Coulais \& Abergel 2000). Each data set was then examined carefully, frame by frame, and further bad pixels were removed manually as required. Most of these occurred in regions immediately adjacent to pixels which had been automatically de-glitched. An approximate criterion for deglitching was if the pixel value exceeded $3 \sigma$ of the average of the 5 frames that surrounded it in time. In addition, although the main effects of memory had been removed as indicated above, the detector still retained a weak memory of the galaxy emission in the first frames of any given off-source sky field. Therefore, the first $\approx 15$ frames from sky fields were also removed, as were distinct bright objects in the sky fields. 
Table 2. Galaxy group membership ${ }^{1}$.

\begin{tabular}{|c|c|c|c|c|c|c|c|}
\hline Galaxy name ${ }^{a}$ & $\begin{array}{l}\text { RA (J2000) } \\
\quad(\text { h m s })\end{array}$ & $\begin{array}{c}\operatorname{Dec}(\mathrm{J} 2000) \\
\left({ }^{\circ},{ }^{\prime \prime}\right)\end{array}$ & $\begin{array}{l}\text { Velocity }^{b} \\
\left(\mathrm{~km} \mathrm{~s}^{-1}\right)\end{array}$ & $\begin{array}{c}\text { Major axis } \\
\text { (arcmin) }\end{array}$ & $\begin{array}{l}\text { Separation } \\
\text { (arcmin) }\end{array}$ & Group ID ${ }^{c}$ & Ref. $^{d}$ \\
\hline NGC 5529 & 141534.1 & 361336 & $2875(32,770)$ & 6.35 & - & GH141, TG86, NOGG773 & $1,2,3$ \\
\hline NGC 5533 & 141607.7 & 352038 & 3866 & 3.1 & 53.4 & GH141, TG86 & 1,2 \\
\hline NGC 5544 & 141702.5 & 363418 & 3040 & 1.08 & 27.3 & GH141, TG86, NOGG773 & $1,2,3$ \\
\hline NGC 5545 & 141705.2 & 363431 & 3079 & 1.3 & 27.8 & GH141, TG86, NOGG773 & $1,2,3$ \\
\hline NGC 5557 & 141825.7 & 362937 & 3213 & 2.3 & 38.1 & GH141, TG86 & 1,2 \\
\hline NGC $5589^{e}$ & 142125.1 & 351614 & 3397 & 1.09 & 91.4 & GH141 & 1 \\
\hline NGC $5590^{f}$ & 142138.4 & 351218 & 3221 & 1.8 & 96.1 & GH141, TG86 & 1,2 \\
\hline NGC 5596 & 142228.7 & 370720 & 3122 & 1.13 & 99.0 & GH141 & 1 \\
\hline NGC 5614 & 142407.6 & 345132 & 3892 & 2.5 & 132.8 & GH141, TG 86 & 1,2 \\
\hline NGC 5656 & 143025.5 & 351915 & 3163 & 1.66 & 188.8 & GH141 & 1 \\
\hline NGC 5675 & 143239.8 & 361808 & 3973 & 2.12 & 206.8 & GH141 & 1 \\
\hline NGC 5684 & 143550.2 & 363236 & 4082 & 1.56 & 245.5 & GH141 & 1 \\
\hline NGC 5695 & 143722.1 & 363404 & 4225 & 1.3 & 264.0 & GH141 & 1 \\
\hline $\mathrm{MCG}+06-31-085 \mathrm{a}$ & 141519.1 & 361208 & 2968 & 0.47 & 3.3 & & 4 \\
\hline Kregel B ${ }^{g}$ & 141535.5 & 361202 & $2959(447)$ & & 1.6 & & 4 \\
\hline \multicolumn{8}{|c|}{ NEW COMPANIONS } \\
\hline $\begin{array}{l}\text { IKPM } 1 \\
\text { J141543.48+361319.1 }\end{array}$ & 141543.5 & 361319 & 3038 & 0.21 & 1.9 & & this work \\
\hline $\begin{array}{l}\text { IKPM } 2 \\
\text { J141600.64+361710.4 }\end{array}$ & 141600.6 & 361710 & 2645 & 0.50 & 6.4 & & this work \\
\hline
\end{tabular}

${ }^{1}$ Data from NED unless otherwise indicated. ${ }^{a}$ Names beginning with $\mathrm{J}$ are identifiers from the SDSS DR5. Those beginning with I are our labels.

${ }^{b}$ Heliocentric. Values in parentheses have been computed from SDSS photometric redshifts. ${ }^{c}$ Group Membership. GH $=$ Geller \& Huchra, TG $=$ Turner \& Gott, NOGG $=$ Nearby Optical Galaxies Group member (hierarchical algorithm). ${ }^{d}$ Reference for Group Membership or association with NGC 5529. 1: van Driel et al. (2001), 2: Turner \& Gott (1976), 3: Giuricin et al. (2000) 4: Kregel et al. (2004b). ${ }^{e}$ Also listed as NGC 5588.

${ }^{f}$ Also listed as NGC 5580. ${ }^{g}$ Position and velocity from Ref. 4 (see $e$ ).

Table 3. Observing and map parameters.

\begin{tabular}{|c|c|c|c|c|}
\hline Parameter & TDT 56100405 ${ }^{a}$ & ${\text { TDT } 56300315^{a}}^{a}$ & $\overline{\text { TDT 56401011 }}^{a}$ & Final map \\
\hline Central wavelength $^{b}[$ Range] $(\mu \mathrm{m})$ & $6.75[5.0-8.5]$ & $6.75[5.0-8.5]$ & $6.75[5.0-8.5]$ & $6.75[5.0-8.5]$ \\
\hline Date of Observations & 30/May/1997 & 01/June/1997 & 02/June/1997 & \\
\hline Observing mode ${ }^{b c}$ & CAM03 & CAM03 & CAM03 & CAM03 \\
\hline Pixel field of view ${ }^{d}(\operatorname{arcsec})$ & 6.0 & 6.0 & 6.0 & 6.0 \\
\hline $\operatorname{PSF}(F W H M)^{e}(\operatorname{arcsec})$ & 7.2 & 7.2 & 7.2 & 7.2 \\
\hline Frame field of view ${ }^{f}$ (arcmin) & 3.2 & 3.2 & 3.2 & 3.2 \\
\hline No. of frames ${ }^{g}$ & 675 & 675 & 675 & \\
\hline Integration time per frame (s) & 10.08 & 10.08 & 10.08 & \\
\hline Calibration error ${ }^{b}(\%)$ & 3.3 & 3.3 & 3.3 & \\
\hline $\bar{\sigma}, \mathrm{rms}_{\sigma}{ }^{h}\left(\mathrm{mJy}\right.$ pixel $\left.^{-1}\right)$ & $0.058,0.083$ & $0.035,0.026$ & $0.032,0.030$ & $0.044,0.039$ \\
\hline $\begin{array}{l}\text { Peak map flux }\left(\mathrm{mJy} \mathrm{pixel}^{-1}\right) \\
\left(\mathrm{mJy} \operatorname{arcsec}^{-2}\right)\end{array}$ & 6.50 & 7.57 & 8.23 & $\begin{array}{c}7.43 \\
0.203\end{array}$ \\
\hline $\begin{array}{l}\operatorname{Rms}^{i}\left(\mathrm{mJy} \mathrm{pixel}^{-1}\right) \\
\quad\left(\mathrm{mJy} \mathrm{arcsec}^{-2}\right)\end{array}$ & 0.020 & 0.021 & 0.033 & $\begin{array}{c}0.014 \\
0.0003\end{array}$ \\
\hline Dynamic Range $^{j}$ & 325 & 360 & 249 & 531 \\
\hline
\end{tabular}

${ }^{a}$ TDT No. is a unique number that identifies the ISO observation. ${ }^{b}$ Blommaert et al. (2003). ${ }^{c}$ CAM03 is a beam-switching mode for photometric imaging. ${ }^{d}$ Size of a square pixel on the sky. ${ }^{e}$ Full width at half maximum of the point spread function. ${ }^{f}$ Size of the square frame which contains an array of $32 \times 32$ pixels. The final image size is slightly smaller due to editing. ${ }^{g}$ Number of frames in the data set. Approximately $40 \%$ of these frames were on-source. ${ }^{h}$ Average $\sigma$ and rms in $\sigma$, respectively, over the field, where the $\sigma$ is the formal random error carried through by the software throughout the data reductions. A typical error in a single pixel value would be given by $\bar{\sigma}{ }^{i}$ Measured rms noise level of the map. ${ }^{j}$ Peak map flux divided by rms.

The on-source frames were then averaged and corrected for the flat field, its distortions and sky brightness using the offsource sky frames. The result was calibrated to $\mathrm{mJy} / \mathrm{pixel}$ units following Blommaert et al. (2003). Formal errors were also carried through the data reduction on a pixel-by-pixel basis, following the description in Irwin \& Madden (2006). The parameters of the individual maps and their corresponding error maps are provided in Table 3.

Each image and its corresponding error map was then read into the Astronomical Image Processing System (AIPS) and subsequent analysis carried out in this package. The 3 images were compared for consistency in position and flux. At this point, the second data set required a slight positional adjustment (2.5 pixels in RA) to bring it into alignment with the other two images. We also interpolated over a known bad pixel column. The three images were then averaged to form a final map, retaining the original 6 " pixel size, and the same procedure was then followed for the error maps. The parameters of the averaged map and averaged error map are also given in Table 3. Note that $\bar{\sigma}$ represents a typical formal error in any given pixel, whereas 
Table 4. Off-source pointing positions.

\begin{tabular}{cc}
\hline \hline $\begin{array}{c}\text { Right ascension (J2000) } \\
(\mathrm{h} \mathrm{m} \mathrm{s})\end{array}$ & $\begin{array}{c}\text { Declination }(\mathrm{J} 2000) \\
\left({ }^{\circ},{ }^{\prime \prime}\right)\end{array}$ \\
\hline 141536.10 & 361834.8 \\
141532.65 & 362316.8 \\
141535.47 & 362215.9 \\
141534.87 & 362108.3 \\
141531.95 & 361555.6 \\
141535.34 & 361418.3 \\
141535.64 & 361410.5 \\
141532.87 & 361002.9 \\
141533.74 & 360644.9 \\
141536.27 & 360758.0 \\
141533.69 & 361433.9 \\
141534.15 & 361442.3 \\
141536.49 & 361413.0 \\
141600.34 & 361441.6 \\
141612.24 & 361525.2 \\
141615.29 & 361419.5 \\
141604.60 & 361439.8 \\
141534.08 & 361529.0 \\
141534.03 & 361441.1 \\
141534.08 & 361418.0 \\
141504.45 & 361516.0 \\
141451.92 & 361517.1 \\
141459.43 & 361413.9 \\
141526.41 & 361501.3 \\
141532.83 & 361512.4 \\
141535.66 & 361412.9 \\
\hline
\end{tabular}

the rms, which is lower, is measured directly from the image and represents pixel-to-pixel variations in noise across the map. The latter value has been significantly reduced by combining the three data sets into one.

The averaged map and its error map were then interpolated onto a $1^{\prime \prime}$ square pixel grid and rotated to the correct orientation on the sky. The resulting ISO and error maps are shown in Fig. 2. A final positional shift of less than a pixel of the original data (3.4" in RA and 5.1" in Dec) was then applied to these maps to align the ISO map with the SDSS $r$-band image. The fine adjustment in astrometry was possible because of two sources, shown with crosses in the overlay of Fig. 3, that are visible in both the ISO and optical images. Characteristic map errors are also listed in Table 3. In addition, there is an absolute calibration error of order, $\pm 15-20 \%$ (e.g. Coia et al. 2005; Pagani et al. 2003), that will affect comparisons with images at other wavelengths, but should not affect point-to-point comparisons on the ISO map itself.

\section{Results}

Figure 2 shows the final ISO $\lambda 6.75 \mu \mathrm{m}$ emission along with its error map, and an overlay of the emission on the SDSS $r$-band image is shown in Fig. 3. Before studying these data in detail, however, it is important to establish whether there might be other significant contributors to the $\lambda 6.75 \mu \mathrm{m}$ emission, besides PAHs. These might include an underlying dust continuum ${ }^{3}$, and a stellar continuum from cool stars.

3 In this paper, we distinguish the PAH carriers as those components that form spectral features and refer to an underlying continuum as a possible contribution from dust whose modified black body emission increases with increasing wavelength at $\lambda 6.75 \mu \mathrm{m}$ (e.g. Fig. 7 , inset, of Irwin \& Madden 2006). Such a continuum may be due to very small grains (VSGs, Cesarsky et al. 2000).

\subsection{The $\lambda 6.75 \mu \mathrm{m}$ emission}

Previous observations and spectral modeling indicate that the MIR spectrum of spiral galaxies is overwhelmingly dominated by PAH emission (e.g. Smith et al. 2007; Draine \& Li 2007; Vogler et al. 2005; Lu et al. 2003; Genzel \& Cesarksky 2000, and references therein) and, as indicated in Sect. 1, the $\lambda 6.75 \mu \mathrm{m}$ ISO band is no exception. Within this band fall the $\lambda 6.2 \mu \mathrm{m}$, $\lambda 7.7 \mu \mathrm{m}$, and the short wavelength wing of the $\lambda 8.6 \mu \mathrm{m} \mathrm{PAH}$ emission features. Of these, the $\lambda 7.7 \mu \mathrm{m}$ feature is the strongest (see Fig. 7 of Irwin \& Madden 2006) and can be modeled as a blend of three spectral features at $\lambda 7.42 \mu \mathrm{m}, \lambda 7.60 \mu \mathrm{m}$, and $\lambda 7.85 \mu \mathrm{m}$ (Smith et al. 2007). The $\lambda 7.7 \mu \mathrm{m}$ PAH complex, alone, can contribute nearly one-half of the total PAH luminosity and up to $10 \%$ of the total infrared luminosity (Smith et al. 2007). Indeed, photometry of the $\lambda 6.75 \mu \mathrm{m}$ ISO band is normally taken as a direct proxy for the presence of PAH emission.

It has also become clear that galaxies show qualitatively little difference in their MIR spectra and that spectral shape is largely independent of star formation rate (Vogler et al. 2005; Lu et al. 2003; Genzel \& Cesarsky 2000, and references therein). Lu et al. (2003), for example, found band-to-band variations of, at most, $15 \%$ in the mid-IR spectrum out to $\lambda 11 \mu \mathrm{m}$. It is now apparent, however, that some variations in $\mathrm{PAH}$ line ratios can exist, depending on their environment. The ratios between the $\lambda 6.2$, $\lambda 7.7$, and $\lambda 8.6 \mu \mathrm{m}$ PAHs do not seem to show much variation within galaxies and between galaxies (Galliano et al. 2007), but the $\lambda 6.2 / \lambda 11.3$ and $\lambda 7.7 / \lambda 11.3$ ratios can vary by up to an order of magnitude (Lu et al. 2003; Vogler et al. 2005; Draine \& Li 2007; Smith et al. 2007; Galliano et al. 2007). This variation can be controlled by the fraction of ionized PAHs, which has been demonstrated to be linked to $G_{0} / n_{\mathrm{e}}$ (the ratio of the intensity of the UV radiation field to the electron density, Galliano et al. 2007) or to the modification of the grain size distribution (Smith et al. 2007). For this study, since we are dealing only with the $\lambda 6.75 \mu \mathrm{m}$ ISOCAM band, we can assume constant band ratios between the $\lambda 6.2, \lambda 7.7$ and $\lambda 8.6 \mu \mathrm{m}$ PAH features, although some variation will not affect our conclusions.

As for an underlying hot dust continuum, such a contribution has been shown to be negligible in normal star forming galaxies (e.g. Galliano et al. 2007). For example, the three PAH features at $\lambda 6.2, \lambda 7.7$, and $\lambda 8.6 \mu \mathrm{m}$ in M 82 can be fit without any significant underlying continuum at all (Laurent et al. 2000). Vogler et al. (2005) also find that a continuum contributes only $\sim 5 \%$ for M 83 in the ISO bands that trace PAH emission. Depending on the assumption of PAH line profile, recent models of MIR spectral energy distributions (SEDs, e.g. Draine \& Li 2007; Galliano et al. 2007) typically show an underlying continuum that is at least an order of magnitude lower than the PAH spectral features.

A stellar component, on the other hand, may be a somewhat larger contributor to the observed emission. Spitzer Space Telescope observations, for example, are typically corrected for a stellar component based on the $\lambda 3.6 \mu \mathrm{m}$ image which is assumed to contain only stellar emission. The correction is based on an extrapolation of the stellar flux to the relevant wavebands according to the prescription of Helou et al. (2004) who have used stellar population models and model photospheric SEDs derived from Starburst99 (Leitherer et al. 1999). For NGC 5529, the 2 MASS $K_{\mathrm{s}}$ band $(\lambda 2.16 \mu \mathrm{m})$ image is available and we can also assume that this band contains only stellar emission. Using the same relation of Helou et al., we estimate that the global flux 


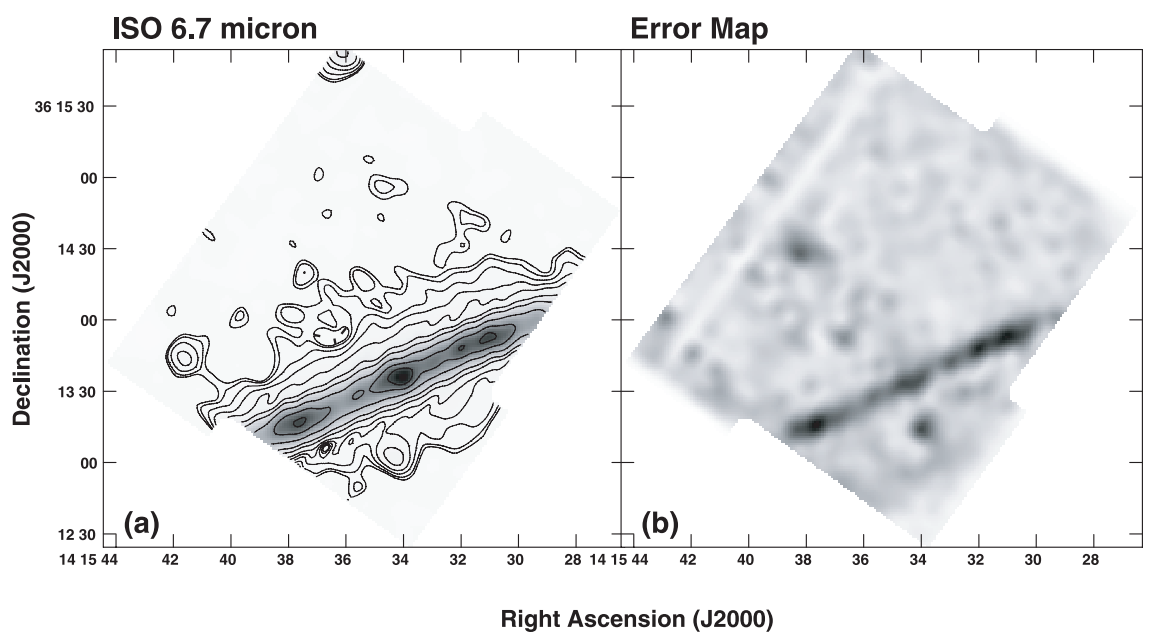

Fig. 2. a) Final ISO $\lambda 6.7 \mu \mathrm{m}$ map of NGC 5529 in both contours and greyscale. The greyscale is over a linear range from -0.005 to $0.200 \mathrm{mJy} \operatorname{arcsec}^{-2}$ and the truncated boundary shows the region of sky that was mapped. Contours are at $0.0006(2 \sigma)$, $0.0009,0.0014,0.0023,0.0040,0.0100,0.0200$, $0.0500, \quad 0.1000$, and $0.1500 \mathrm{mJy} \operatorname{arcsec}^{-2}$. Small tick marks indicate a declining contour. b) Error map, as described in the text, shown in linear greyscale from -0.0001 to $0.008 \mathrm{mJy} \operatorname{arcsec}^{-2}$. The straight white bar stretching from top center to lower left shows the location of the bad column on the detector. of NGC 5529 in the $\lambda 6.75 \mu \mathrm{m}$ band contains a contribution of stellar flux that amounts to $14 \%$ of the 2 MASS $\lambda 2.16 \mu \mathrm{m} \mathrm{flux}^{4}$.

The 2MASS $K_{\mathrm{s}}$ band image, smoothed to the resolution of the ISO $\lambda 6.75 \mu \mathrm{m}$ band and multiplied by $14 \%$ is shown in the Inset to Fig. 3. An integration of this map over the same region as is visible in the $\lambda 6.75 \mu \mathrm{m}$ image indicates that, globally, stellar emission contributes $17 \%$ of the flux in the ISO $\lambda 6.75 \mu \mathrm{m}$ band. This is within the range estimated for the absolute calibration error on typical ISOCAM observations $( \pm 15 \rightarrow 20 \%$, Sect. 3$)$ and therefore is justifiably neglected for the galaxy as a whole. In the region of the halo itself, no stellar halo is observed (Fig. 3 Inset) to the limits of the available 2MASS data. The rms noise of this map places an upper limit of $\sigma_{*}=0.0002 \mathrm{mJy} \mathrm{arcsec}^{-2}$ on the stellar contribution to the $\lambda 6.75 \mu \mathrm{m}$ band, i.e. an upper limit of approximately $30 \%$ on the lowest contour shown in Figs. 2a, 3 (main) and 5 and a lower percentage for the higher contours. In a rectangular region aligned with the major axis centered on the north-east halo ${ }^{5}$ the maximum contribution of the stellar halo is $7 \%$. Since the fraction of stellar emission could vary from location to location, a possible stellar contribution to the halo is discussed further in Sect. 4.3.

\subsection{Disk emission}

Figure 2a shows strong PAH emission along the disk of NGC 5529, although the entire disk has not been mapped due to the truncated field of view. The emission reaches a maximum at the nucleus and a two-dimensional Gaussian fit to this maximum gives a position of $\mathrm{RA}=14^{\mathrm{h}} 15^{\mathrm{m}} 34.1^{\mathrm{s}} \pm 0.2^{\mathrm{s}}$, Dec $=$ $36^{\circ} 13^{\prime} 36^{\prime \prime} \pm 2^{\prime \prime}$, which agrees with the optical center (Table 1). Several other peaks are also observed along the major axis, a double peak $\approx 40^{\prime \prime}$ from the nucleus along the north-west major axis and a single peak $\approx 50^{\prime \prime}$ to the south-east. This structure is shown more readily in the plot of intensity along the major axis shown in Fig. 4a. Note that there is no change to the positions and structure described here if a stellar contribution, as outlined in Sect. 4.1, is subtracted from the map, pixel by pixel.

${ }^{4}$ Note that Irwin \& Madden (2006) incorrectly estimated the stellar flux in their observing bands for NGC 5907. A recalculation, based on the Helou et al. (2004) relation, however, results in corrections of order a few per cent, so their numerical results and conclusions are not changed.

5 The rectangle was set so that it is parallel to the major axis with a lower edge $8 \operatorname{arcsec}(1.7 \mathrm{kpc})$ above the plane in order to start a full beam width from midplane and be outside of the modeled disk (see Sect. 4.3). The rectangle's size is $121 \operatorname{arcsec}(25.8 \mathrm{kpc})$ parallel to the major axis and $61 \operatorname{arcsec}(13.0 \mathrm{kpc})$ parallel to the minor axis.
Irwin \& Madden (2006) found that the PAH emission in NGC 5907 followed primarily the molecular gas distribution in that galaxy, with a fainter wing of emission extending farther out into the HI dominated region. To our knowledge, there is no published CO map of NGC 5529 with which to compare the PAH distribution. The HI distribution (Kregel et al. 2004b) has a hole at the nucleus and the HI emission rises on either side of the nucleus to maxima that are at positions farther out in radius than the peaks of Fig. 4a. Therefore, the HI distribution does not resemble that of the PAHs, to the limits of these measurements. We suspect that the bulk of the emission will follow that of the $\mathrm{CO}$, as in NGC 5907, once such measurements are made.

\subsection{Halo emission}

Figure 2a and especially Fig. 3 show that the PAH emission extends far from the plane of NGC 5529. This detection has been possible because of the high sensitivity and dynamic range (Table 3) of the beam-switching observations. The high-latitude emission shows considerable structure, a result that was also seen for NGC 5907. Indeed, among other features, two discrete arc-like structures can be seen on the north-east side of the major axis: one above the nucleus extending to $\mathrm{RA} \approx 14^{\mathrm{h}} 15^{\mathrm{m}} 36^{\mathrm{s}}$, Dec $\approx 36^{\circ} 14^{\prime} 10^{\prime \prime}$, and one farther to the east extending to RA $\approx$ $14^{\mathrm{h}} 15^{\mathrm{m}} 39^{\mathrm{s}}$, Dec $\approx 36^{\circ} 13^{\prime} 50^{\prime \prime}$. Arc-like features similar to these have been observed in other ISM tracers in other edge-on galaxies (e.g. Lee et al. 2001). At the $3 \sigma$ contour shown in Fig. 3, the emission extends to $z \approx 40^{\prime \prime}(z \approx 8.5 \mathrm{kpc})$ above the plane. Note that there is no emission (Fig. 3 Inset), to the $2 \sigma$ limit of the stellar map, to this height. The maximum extent of the stellar emission at the $2 \sigma$ limit of this map is $z \approx 20^{\prime \prime}(4.2 \mathrm{kpc})$.

Even higher sensitivity can be achieved by averaging the emission over a broad swath of the major axis (91" in width) and plotting the result as a function of latitude, $z$, above and below the plane (note that no correction for inclination is required). Not only does this approach improve the sensitivity, but it also allows us to probe whether a broad scale halo exists around the galaxy, rather than only discrete extensions. The result is shown in Fig. 4b (main figure). Note that the dynamic range of this slice is very high, i.e. 1773/1 (peak/rms). Various functions were fitted to these data including an exponential, a Gaussian, a hyperbolic secant, and the square of a hyperbolic secant (that is, so that the range of functions used by others for optical data (Sect. 2) are also considered here). The best fit was to a simple Gaussian, the result shown as the grey curve in Fig. 4b (main). The fit is excellent, with the exception of the broad scale wings that are seen in 


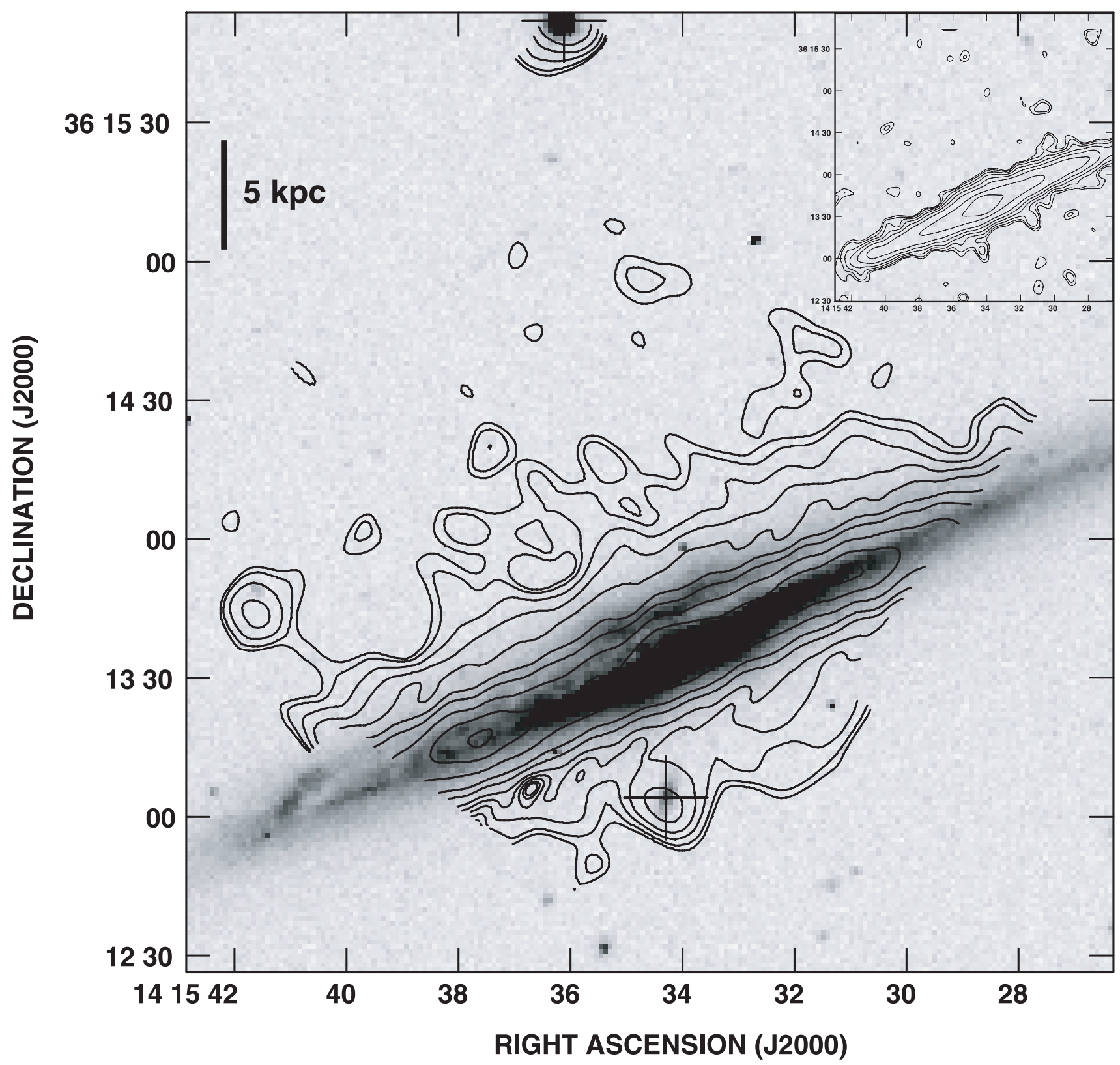

Fig. 3. ISO $\lambda 6.75 \mu \mathrm{m}$ image, shown with the same contours as in Fig. 2a, overlaid on the SDSS $r$-band iamge. Fine adjustments to the astrometry were made using the objects marked with crosses, i.e. the star at the top of the field and the galaxy just below NGC 5529 identified as $\mathrm{J} 141534.3+361304.5$ in the SDSS DR5 (likely a background source with a photometric redshift of $z_{\mathrm{ph}}=0.123$ ). The scale is marked with a vertical bar. Inset: 2MASS $K_{\mathrm{s}}$ band image of NGC 5529, smoothed to the same resolution as the ISO $\lambda 6.75 \mu \mathrm{m}$ image, and multiplied by a factor of 0.14 to represent the approximate strength of the stellar emission at $\lambda 6.75 \mu \mathrm{m}$ (see Sect. 4.1). Contours are the same as for the ISO $\lambda 6.75 \mu \mathrm{m}$ image except for the addition of the lowest contour of $0.0004 \mathrm{mJy} \operatorname{arcsec}^{-2}(2 \sigma)$.

the data but not in the model (see below). The displayed best fit Gaussian curve, which is wider than the true distribution because of beam smoothing, has $\sigma_{\mathrm{G}}=4.6^{\prime \prime}(979 \mathrm{pc})$, or a full width at half maximum of $F W H M=10.8^{\prime \prime}(2.3 \mathrm{kpc})$. We reproduced this curve by modeling the $z$ distribution of the disk as a Gaussian of $F W H M=8.0^{\prime \prime}(1.7 \mathrm{kpc})$ and then convolving with the Gaussian PSF of the observations $\left(F W H M_{\mathrm{PSF}}=7.2^{\prime \prime}\right.$, Table 3$)$. Thus, after beam correction, the bulk of the PAH emission can be represented by a disk that declines as a Gaussian of FWHM $=8.0^{\prime \prime}$ $(1.7 \mathrm{kpc})$, or $\sigma_{\mathrm{G}}=3.4^{\prime \prime}(726 \mathrm{pc})$. We repeated the same exercise over the same region using the $\mathrm{H} \alpha$ data $^{6}$, finding a

6 The $\mathrm{H} \alpha$ data were kindly made available by Scott Miller. Note that Miller \& Vielleux (2003) fit the main disk emission with an exponential;
Gaussian of $F W H M=13.2^{\prime \prime}(2.8 \mathrm{kpc})$, or $\sigma_{\mathrm{G}}=5.6^{\prime \prime}(1.2 \mathrm{kpc})$. Thus, the $\mathrm{H} \alpha$ disk is $\approx 1.6 \times$ thicker than the PAH disk, when the same regions and criteria are used.

The faint wings of PAH emission in the very high sensitivity plot of Fig. 4b (main) reveal that, as suggested by the appearance of Figs. 2a and 3, a broad scale halo of PAH emission exists around NGC 5529. The Inset to Fig. 4b shows the same averaged data from the main figure, but over the north-east side of the disk only, to avoid contamination by the small galaxy (likely a background object with $z_{\mathrm{ph}}=0.123$ ) on the south-west side. To the $3 \sigma$ limit of the averaged minor axis slice (shown by the short

we have repeated our analysis on their data so as to directly compare best-fit Gaussians. 

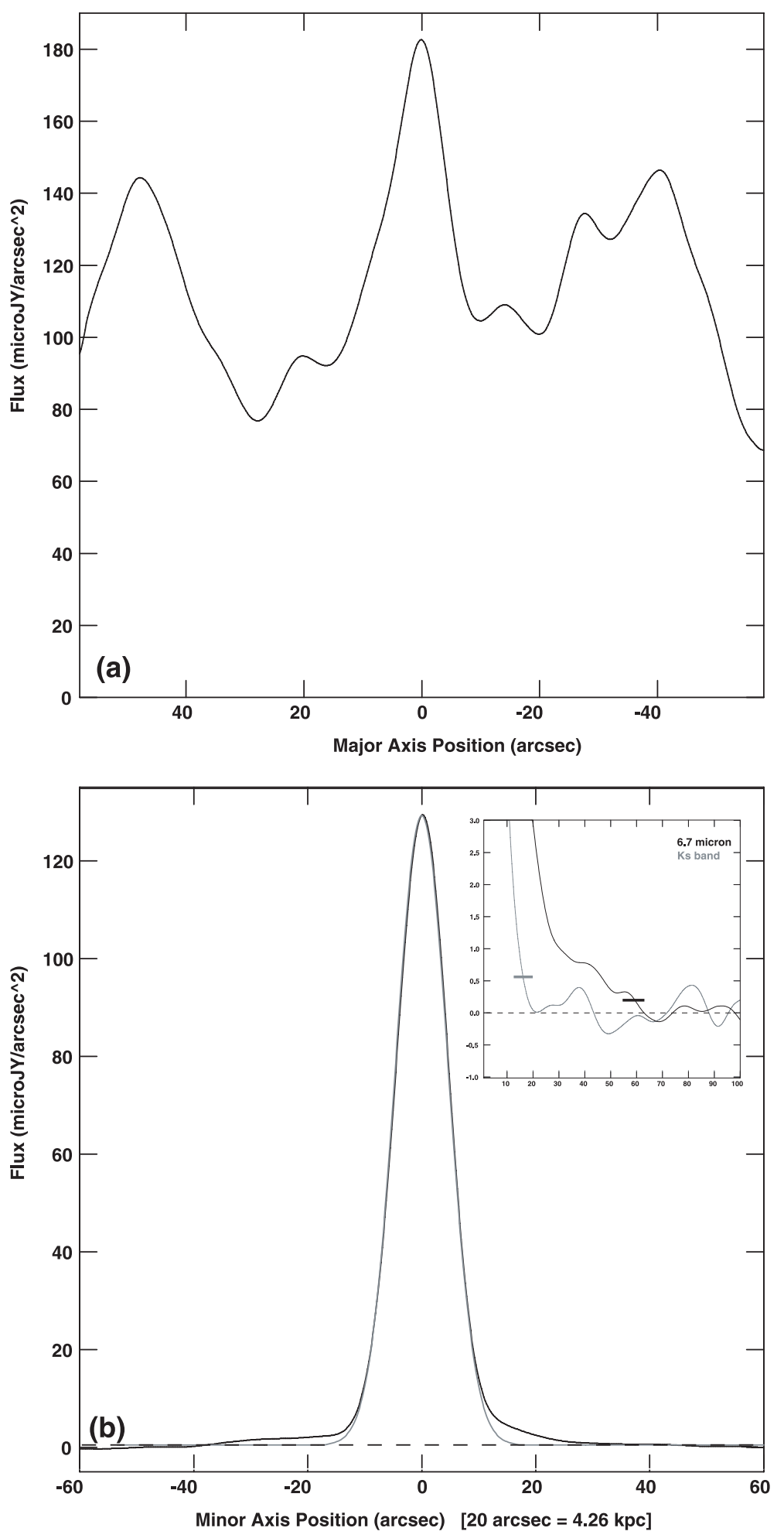

Fig. 4. a) Intensity distribution of the major axis of NGC 5529, averaged over a strip centered at mid-plane and of vertical width, $7^{\prime \prime}$. The center is at the galaxy's nucleus and positive values correspond to the south-east side of the galaxy. b) Intensity distribution along the minor axis of NGC 5529, averaged over a strip centered at the galaxy's center and of width, $91^{\prime \prime}$, along the major axis. The dynamic range of this slice is 1773/1. Positive values correspond to the north-east side of the galaxy's disk. The grey curve is the best fit Gaussian (see text). (Inset) Blowup of the minor axis distribution in the direction towards the north-east only (black curve). The axes have the same units as the larger figure. The gray curve is the minor axis slice of the $K_{\mathrm{s}}$-band image shown in the Inset to Fig. 3 averaged over the same region as the $\lambda 6.7 \mu \mathrm{m}$ image for comparison. The two short horizontal bars mark the $3 \sigma$ levels of the $\lambda 6.7 \mu \mathrm{m}$ minor axis slice (black, $1 \sigma=0.073 \mu \mathrm{Jy} \operatorname{arcsec}^{-2}$ ) and $K_{\mathrm{s}}$-band slice (grey, $1 \sigma=0.18 \mu \mathrm{Jy} \operatorname{arcsec}^{-2}$ ), respectively. horizontal bar), emission is seen out to $z \approx 60^{\prime \prime}(z \approx 12.8 \mathrm{kpc})$. After subtracting the modeled Gaussian of the prominent disk emission, the residual wing emission can be fit with an exponential of $z_{\mathrm{e}} \approx 17.5^{\prime \prime}$ (3.7 kpc). Miller \& Veilleux (2003) found an exponential fit of $z_{\mathrm{e}}=4.5 \mathrm{kpc}$ for the high latitude wings of the $\mathrm{H} \alpha$ emission, i.e. the scale height of the high latitude $\mathrm{H} \alpha$ emission is $\approx 1.2 \times$ larger than that of the PAH emission.

Could the extended $\lambda 6.7 \mu \mathrm{m}$ emission be explained in some other way than a PAH halo? As indicated in Sect. 4.1, the global contribution of stellar emission is within the absolute calibration error of the $\lambda 6.7 \mu \mathrm{m}$ band emission, if standard extrapolations are reliable. To further compare the halo stellar emission with that of the $\lambda 6.7 \mu \mathrm{m}$ band, we repeat the same averaging technique to the $K_{\mathrm{s}}$ band image of Fig. 3 (Inset) and we show the resulting stellar emission profile in Fig. $4 \mathrm{~b}$ (Inset, grey curve). No emission above the $3 \sigma$ level of this plot is seen beyond $z \approx 20^{\prime \prime}$ (short horizontal grey line) in contrast to $z \approx 60^{\prime \prime}$ seen at $\lambda 6.7 \mu \mathrm{m}$. Similar results are obtained when the SDSS $i$ and $z$-band images are examined in the same way (slices not shown). Even if we allow the $K_{\mathrm{s}}$ band emission to contribute to 


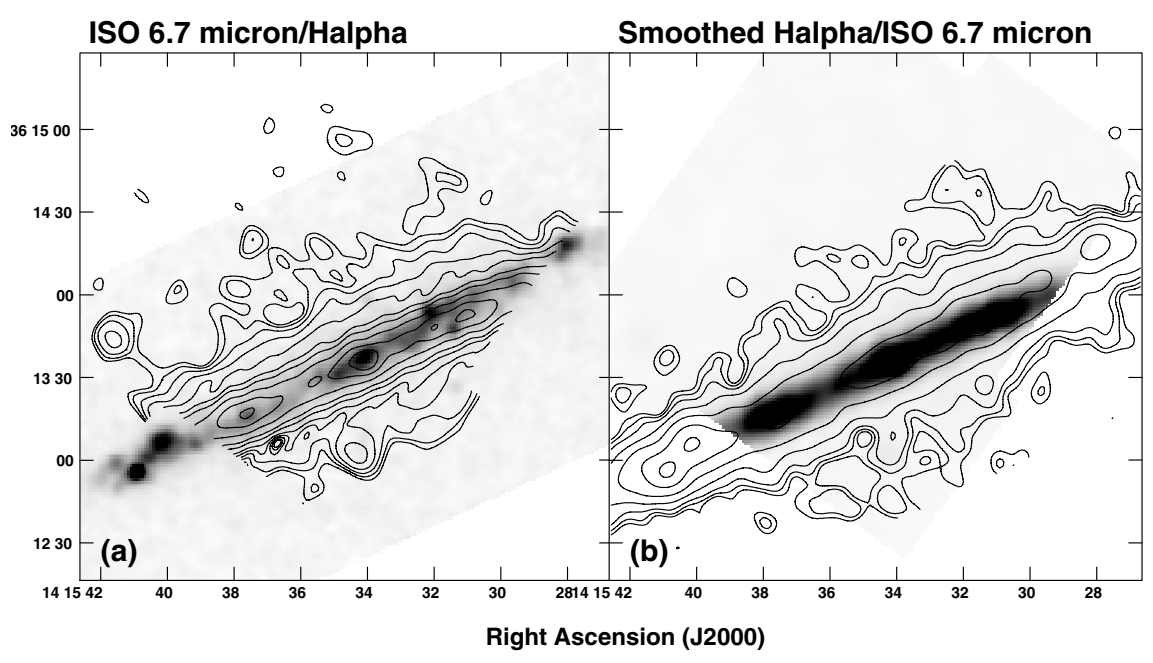

Fig. 5. a) ISO $\lambda 6.7 \mu \mathrm{m}$ image, shown with the same contours as in Fig. 2a, overlaid on a linear greyscale $\mathrm{H} \alpha$ image of Miller \& Veilleux (2003). The $\mathrm{H} \alpha$ image is in arbitrary units and is shown to emphasize detail in the disk. b) Here, the contours (arbitrary units) are of $\mathrm{H} \alpha$ emission, smoothed to $7.2^{\prime \prime}$ resolution to emphasize high latitude emission, overlaid on the ISO $\lambda 6.7 \mu \mathrm{m}$ image in linear greyscale (greyscale range $=-0.003$ to $\left.0.10 \mathrm{mJy} / \operatorname{arcsec}^{-2}\right)$. the wings at approximately a $1 \sigma$ level over the entire wing extent, stellar emission could not account for the $\lambda 6.7 \mu \mathrm{m}$ halo. However, taking this conservative approach, the maximum extent of the halo then adjusts to $z \approx 50^{\prime \prime}(z \approx 10.6 \mathrm{kpc})$. Finally, we note that the PSF is known to be Gaussian to high accuracy (see Galliano 2004; and Irwin \& Madden 2006) in this ISO band and the extended PAH emission cannot be explained by PSF emission wings.

In summary, a significant halo of PAH emission is seen around NGC 5529 and shows considerable substructure with some features that are vertical or arc-like with respect to the disk. The bulk of the emission in the vertical $z$ direction can be fit by a gaussian with dispersion, $\sigma_{\mathrm{G}}=3.4^{\prime \prime}(726 \mathrm{pc})$ and, after subtracting this main emission, faint PAH wings are seen with an exponential vertical scale height of $z_{\mathrm{e}}=17.5^{\prime \prime}(3.7 \mathrm{kpc})$. To the $3 \sigma$ limit of the data and allowing for a small contribution from stars, emission is seen as far out as $z=50^{\prime \prime}(10.6 \mathrm{kpc})$. This is an exceptional distance from the plane, exceeding that of $z \approx 6.5 \mathrm{kpc}$ found for NGC 5907 (Irwin \& Madden 2006).

\subsection{PAH $-\mathrm{H} \alpha$ comparison}

As indicated in Sect. 2, the only previously-observed gaseous halo in NGC 5529 was detected by Miller \& Veilleux (2003) in $\mathrm{H} \alpha$ emission, and we have shown in Sect. 4.3 that the vertical distributions in both these bands can be fit with two vertical components, a narrower Gaussian containing most of the emission, and fainter exponential wings that extend much farther. The $\mathrm{H} \alpha$ scale heights are, on average, $\approx 1.4$ times larger than those of the PAHs when global halo emission is considered. In this section, we wish to investigate a possible spatial correlation between the $\mathrm{H} \alpha$ and PAH band emission. This is shown in the two overlays of Fig. 5.

In Fig. 5a, the $\mathrm{H} \alpha$ emission is shown in greyscale with in-disk emission emphasized in order to discern whether the high-latitude PAH emission may be related to underlying indisk emission. The comparison is not straight-forward since the observed halo structure results from an integration of emission along lines-of-sight that vary with radius, the $\mathrm{H} \alpha$ emission in the disk suffers from extinction, and the spatial resolutions are different. The arc (see Sect. 4.3) that, in projection, is located above the nucleus is possibly related to enhanced SF activity in the nuclear vicinity, but since the extended halo emission is so pervasive, one-to-one correlations with in-disk activity cannot be pinpointed with certainty from these observations.

Figure $5 \mathrm{~b}$ shows the $\mathrm{H} \alpha$ emission in contours, smoothed to the same resolution as the ISO data, over the ISO emission in greyscale. With the $\mathrm{H} \alpha$ emission smoothed, the $\mathrm{H} \alpha$ halo is very conspicuous and the "filamentary eDIG" on the northeast side of the galaxy noted by Miller \& Veilleux (2003) is now seen with prominent structure that bears a remarkable resemblance to that of the PAH emission. The two PAH halo arcs are also seen in $\mathrm{H} \alpha$, as is an above-disk feature located at $\mathrm{RA} \approx 14^{\mathrm{h}} 15^{\mathrm{m}} 32^{\mathrm{s}}$, Dec $\approx 36^{\circ} 14^{\prime} 40^{\prime \prime}$. Similarities on the south-west side of the disk are also apparent. Thus, although the vertical scale heights of the $\mathrm{H} \alpha$ emission exceed those of the PAHs (Sect. 4.3), there appears to be a spatial correlation between the PAH halo structure and that of the $\mathrm{H} \alpha$-emitting eDIG in NGC 5529. To properly quantify such a correlation requires a three-dimensional model of the two components and the low $\mathrm{S} / \mathrm{N}$ of the data in the halo region does not support such an approach. However, to "zeroth order", we have investigated whether the two maps are correlated in the region of the north-east halo. After applying a $2 \sigma$ cutoff to both maps, the resulting halo emission spans a projected area of 0.79 square arcminutes. For this region, we computed cross-correlation coefficients between the two maps, finding a best value of $95 \%$ for zero shift in position. This confirms that there is a correlation between the $\mathrm{PAH}$ and $\mathrm{H} \alpha$ halo emission in NGC 5529.

\section{Discussion}

The presence of a large-scale, structured PAH halo about NGC 5529 is a significant result of these observations. Few statistics exist on the presence of PAHs in galaxy halos. Of normal star forming galaxies or those of low SFR, NGC 5529 and NGC 5907 appear to be the only known examples, thus far (see Table 1). Tacconi-Garman et al. (2005) have found a $\lambda 3.3 \mu \mathrm{m}$ PAH feature in the superwind of the starburst galaxy, NGC 253, with $z$ extent $<120 \mathrm{pc}$, and there is now a clear PAH signature in the halo and superwind of M 82 to a distance of $6 \mathrm{kpc}$ from the plane of that galaxy (Engelbracht et al. 2006). Its $8 \mu \mathrm{m}$ emission, which has a strong PAH component, resembles the $\mathrm{H} \alpha$ emission of the superwind in M 82, indicating that PAHs can survive in such an energetic environment and providing another example of a galaxy in which PAHs correlate with $\mathrm{H} \alpha$ emission in the 
halo. Engelbracht et al. also find that filamentary $8 \mu \mathrm{m}$ emission is present, not only in the superwind outflow, but also throughout the halo region, as we see for NGC 5529. They attribute the widespread halo PAHs to previous activity related to star formation. Thus halo PAHs are now seen in galaxies with both high and low SFRs.

PAHs are believed to originate in the dusty envelopes of evolved AGB stars (e.g. Boersma et al. 2006; Matsuura et al. 2004; Cherchneff 1995) and there is now also good evidence that PAHs may be formed from the destruction of small grains (Cesarsky et al. 2000; Rapacioli et al. 2005; Berné et al. 2007). Indeed, PAHs which have fewer than $5.75 \times 10^{4}$ carbon atoms have radii less than $0.005 \mu \mathrm{m}$ (Draine \& Li 2007) and are therefore similar in size to the very small grains (VSGs) that were first introduced to explain the $12 \mu \mathrm{m}$ diffuse emission seen in the Milky Way.

In either case, PAHs are expected to reside in galaxy disks, rather than their halos, unless a distribution of halo AGB stars were the origin of the PAHs. We have insufficient theoretical and observational data to quantify the latter possibility ${ }^{7}$ but we note that the presence of structured, vertical and arc-like features connected to the disk in both NGC 5907 and NGC 5529 argue that halo PAHs originate in the underlying galaxy disk and are ejected into the halo. We expect, for example, that an origin in halo AGB stars would result in a more uniform distribution of halo emission. Furthermore, for NGC 5529 in particular, the PAH - H $\alpha$ correlation (Sect. 4.4 and below) argues for a disk origin since it is widely believed, based on correlations with disk SFRs for example, that the eDIG in galaxies originates in the in-disk ISM. We therefore assume that the halo PAHs of NGC 5529 originate in its disk, in agreement with Engelbracht et al. who similarly argue for a disk origin for the halo PAHs of M 82 that are unrelated to the nuclear outflow.

In the Milky Way, we know that PAH emission tends to be strongest in photodissociation regions (PDRs) since, in such regions, there are sufficient far-UV (FUV) photons to excite the PAHs but not so many that the probability of photo-destruction becomes high (see below). For example, the Orion bar PDR shows a strong PAH signature (e.g. Sloan et al. 1997) and shells of PAH emission can be seen around HII regions in the star forming complex, NGC 6334 (Burton et al. 2000). In HII regions, themselves, PAH emission is suppressed because $\mathrm{PAH}$ molecules cannot survive for long in such a harsh environment (e.g. Dopita et al. 2005). On the other hand, some PAH emission has indeed been observed in ionized regions such as planetary nebulae and HII regions (e.g. Peeters et al. 2005; van Diedenhoven et al. 2004; Vijh et al. 2004).

The survival and emission properties of PAHs in various interstellar environments has now been modeled by a number of authors (Allain et al. 1996a,b; Le Page et al. 2001, 2003; Li \& Draine 2001; Draine \& Li 2007). The most relevant process, when considering the survival of PAHs in galaxy halos, is the photo-destruction rate (we do not consider the possible growth of PAHs through accretion of carbon atoms in the halo environment). Other destruction processes, such as collisional

\footnotetext{
7 To quantify this requires a knowledge of the halo AGB star population, a knowledge of the PAH formation rate (metallicity-dependent) and destruction rate, a SED model that predicts the strength of the various PAH features taking into account stochastic single-photon excitation from in-disk photons (dependent on vertical optical depth), and some indication as to how these quantities vary with time. See Temi et al. (2007a,b) for a similar approach, but for the different conditions in elliptical galaxies, for classical grains rather than PAHs, and assuming dominant destruction by spallation in a hot X-ray environment.
}

destruction in shocks or sputtering from the presence of hot Xray emitting gas seem less likely, the former because of the halo environment, and the latter because no X-ray halo has yet been observed in this galaxy (Sect. 1). PAHs can be photo-dissociated by the loss of a hydrogen atom, a carbon atom, $\mathrm{C}_{2} \mathrm{H}_{2}$, or $\mathrm{H}_{2}$. The type and rate of photo-destruction is a function of $\mathrm{PAH}$ size (i.e. number of carbon atoms, $N_{\mathrm{C}}$ ) and the strength and energy of the radiation field. Large PAHs are more stable than smaller PAHs, in general, and the loss of each particular particle has a different threshold energy for ejection, typical values being in the FUV range, 2 to $8 \mathrm{eV}$.

The lifetime of a large PAH against photo-ejection of particle, $i$, can be estimated from,

$$
\tau=\frac{1}{\chi k_{\mathrm{ISM}}^{i}}
$$

(adapted from Allain et al. 1996a), where $k_{\text {ISM }}^{i}\left(\mathrm{~s}^{-1}\right)$ is the rate of PAH destruction by the ejection of particle, $i$, in the Milky Way's ISM and $\chi$ is a factor that accounts for the difference in the strength of the radiation field between the ISM of the Milky Way and the environment of interest. The rate, $k_{\mathrm{ISM}}^{i}$, is a function of $N_{\mathrm{C}}$ as well as the threshold photon energy for the ejection of species, $i$. For $N_{\mathrm{C}}=50$, for example, $k_{\mathrm{ISM}}^{\mathrm{C}_{2} \mathrm{H}_{2}}=3.56 \times 10^{-18} \mathrm{~s}^{-1}$. The highest destruction rate for $N_{\mathrm{C}}=50 \mathrm{can}$ be attributed to the ejection of $\mathrm{H}$, for which $k_{\mathrm{ISM}}^{\mathrm{H}}=2.3 \times 10^{-16} \mathrm{~s}^{-1}$ (see Allain et al. 1996a). For the halo of NGC 5529, we can set a lower limit to the lifetime of a 50 carbon atom PAH by assuming that the radiation field is as strong as the ISM in the Milky Way $(\chi=1)$ and that destruction is via $\mathrm{H}$ ejection. The resulting lifetime is $\tau=1.4 \times$ $10^{8} \mathrm{yr}$. Thus, even for an unrealistically strong radiation field, such a PAH would be very long lived. Assuming a more realistic halo radiation field in which FUV photons leak from the disk into the halo (adopting $\chi=0.1$ as a possible example) then $\tau=$ $1.4 \times 10^{9}$ yr. In addition, some potentially photo-dissociating FUV photons will instead be absorbed by hydrogen (see, e.g. Dopita 2003, 2005), leading to a longer lifetime. Therefore, we expect halo PAHs of this size to be stable over long time periods. Note, however, that we have not considered any re-settling of PAHs back onto the disk as might occur from a galactic fountain.

We can also ask what vertical velocity, $v_{z}$, would be required in order to eject PAHs from the disk to the halo of NGC 5529 to a height of $\approx 10 \mathrm{kpc}$ (Sect. 4.3) before the PAHs would be photo-dissociated. In the strong radiation field limit $\left(\tau=1.4 \times 10^{8}\right.$ years) the result is $v_{z} \approx 70 \mathrm{~km} \mathrm{~s}^{-1}$. For the more realistic weaker radiation field $\left(\tau=1.4 \times 10^{9}\right.$ years $)$, $v_{z} \approx 7 \mathrm{~km} \mathrm{~s}^{-1}$ is sufficient. The latter value is too low to actually eject material into the halo, but the main conclusion is that PAHs do not need to be supplied into the halo at high velocities in order to account for the observed emission at high galactic latitudes. Since PAHs are long-lived, it is only necessary that there has been sufficient energy to eject the particle into the halo, either at some point in the past or more quiescently over a period of time. This is consistent with the fact that we see PAHs in the halos of galaxies with low SFRs. Moreover, if this is correct, then most star forming galaxies should show some PAH halo emission. The presence of vertical structures (or vertical filamentary features, as described for M 82) suggests that the ejection in NGC 5529 likely did not occur more than $\approx 10^{8}$ years ago; otherwise, galactic rotation would destroy these structures. The process by which PAHs are transported to such high galactic latitudes needs to be more fully addressed by theory and observation. We know that stellar winds and supernovae can contribute to vertical outflows in galaxies and that the outflows will include entrained ISM material. Vertical magnetic fields can also play an important role in 
more quiescent ejection of ISM material. Just how PAHs, either neutral or ionized, might couple to the outflow in either case is not yet clear. In the case of NGC 5529, its interaction with one or more companions may also have played a role in agitating the ISM.

Stellar photons over a range of energy are likely leaking from the disk into the halo regions. The lower energy optical and FUV photons will excite the PAH emission, resulting in the observed MIR bands. Some FUV photons of sufficient energy may also result in photo-dissociation, but at a slow rate (dependent on $N_{\mathrm{C}}$ ). Photons with energies that are higher than $13.6 \mathrm{eV}$ can ionize hydrogen, producing the observed $\mathrm{H} \alpha$ emission. Although, in HII regions in the Milky Way, PAHs are readily destroyed, we note that the radiation field is much stronger in HII regions $(\chi \approx$ $\left.10^{3}\right)$ than in the diffuse ISM $(\chi=1)$ and higher still than in a halo environment (Allain et al. 1996a). Therefore, we would not expect the halo PAHs to avoid $\mathrm{H} \alpha$ emission as they do in the disk ISM. The observed $\mathrm{PAH} / \mathrm{H} \alpha$ correlation in the halo of NGC 5529, then, may simply represent the fact that gas and dust are correlated in the halo (as they are in the disk) and that the sources of excitation (i.e. photons from stars in the disk) are the same.

\section{Conclusions}

NGC 5529 is a large (diameter of $81 \mathrm{kpc})$, edge-on $\left(i=90^{\circ}\right)$ galaxy with a low star formation rate $\left(S F R=3.3 M_{\odot} \mathrm{yr}^{-1}\right)$. It resides in a small galaxy group and we have now consolidated a list of previously known companions from a variety of catalogues. In addition, we identify two new companions, based on their proximity to NGC 5529 and their spectroscopic redshifts, as listed in the Sloan Digital Sky Survey (Data Release 5). In total, there are now at least 17 galaxies in the group, of which NGC 5529 is the dominant member (Table 2).

We have presented ISO beam-switching $\lambda 6.7 \mu \mathrm{m}$ observations of the edge-on galaxy, NGC 5529. These data have very high dynamic range (530/1) and have allowed us to probe both the disk and faint halo region of the galaxy. The $\lambda 6.7 \mu \mathrm{m}$ band is dominated by PAH emission and, globally, it is unlikely that stellar emission constitutes more than $17 \%$ of the total.

The disk PAH emission does not resemble the HI distribution. It peaks at the optical nucleus, whereas the HI distribution has a central hole. Molecular data are lacking, but we expect that the in-disk distribution will follow the molecular gas component as seen in other galaxies. The vertical disk distribution is well fit by a Gaussian with $\sigma_{\mathrm{G}}=726 \mathrm{pc}(F W H M=1.7 \mathrm{kpc})$ after correction for the beam.

In addition, very high latitude PAH emission is also observed, forming a 'halo' around the galaxy with considerable substructure, including vertical features connected to the disk (Fig. 3), arguing for an in-disk origin. The halo PAH emission extends to $z \approx 10 \mathrm{kpc}$, to the limits of the noise on an averaged vertical slice (dynamic range $=1773 / 1$ ). This is the most extensive PAH halo yet observed in a galaxy. The vertical exponential scale height of the extended halo component is $z_{\mathrm{e}}=3.7 \mathrm{kpc}$ which is simewhat smaller than the value of $z_{\mathrm{e}}=4.5 \mathrm{kpc}$ found by Miller \& Vielleux (2003) for the $\mathrm{H} \alpha$ emission in this galaxy. When the $\mathrm{H} \alpha$ distribution is smoothed to the ISO resolution, however, there is a spatial correlation between the $\mathrm{PAH}$ and $\mathrm{H} \alpha$ halo emission.

PAHs can be long-lived in a halo environment which implies that, provided there has been sufficient energy to eject particles into the halo and there are currently sufficient photons leaking from the disk to excite them, a high SFR is likely not necessary for their detection in galaxy halos. Given the very low excitation conditions in galaxy halos in comparison to in-disk HII regions, we do not expect that PAHs will be destroyed in regions in the ionized halo gas as they are in HII regions in the disk. The PAH $\mathrm{H} \alpha$ correlation may result from the fact that both PAHs and ionized components are, to some extent, tracing the actual density distribution in the halo, with excitation from a similar underlying in-disk stellar component. The optical and FUV photons that leak into the halo will excite PAH emission and harder UV photons will ionize the gas.

Acknowledgements. We are grateful to Scott Miller for making the $\mathrm{H} \alpha$ image of NGC 5529 available to us. J.I. would like to thank the Natural Sciences and Engineering Research Council of Canada for her research grant.

\section{References}

Adelman-McCarthy, J. K., et al. 2007, ApJS, submitted

Allain, T., Leach, S., \& Sedlmayr, E. 1996a, A\&A, 305, 602

Allain, T., Leach, S., \& Sedlmayr, E. 1996b, A\&A, 305, 616

Benson, A. J., Bower, R. G., Frenk, C. S., \& White, S. D. M. 2000, MNRAS, 314,557

Berné, O., Joblin, C., Deville, Y., et al. 2007, [arXiv: astro-ph/0703072]

Biviano, A., et al. 1998, The ISOCAM Dark Current Calibration Report, Technical Rep., ESA/ISO Data Centre

Bizyaev, D., \& Mitronova, S. 2002, A\&A, 389, 795

Blommaert, J., Siebenmorgen, R., Coulais, A., et al. 2003, CAM - The Iso Camera, in The ISO Handbook, Vol. II, see

http://www.iso.vilspa.esa.es/users/handbook/

Boersma, C., Hony, S., \& Tielens, A. G. G. M. 2006, A\&A, 447, 213

Brar, R. S., Irwin, J. A., \& Saikia, D. J. 2003, MNRAS, 340, 269

Burton, M. G., Ashley, M. C. B., Marks, R. D., et al. 2000, ApJ, 542, 359

Cesarsky, D., Lequeux, J., Ryter, C., \& Guérin, M. 2000, A\&A, 354, L87

Chanial, P. 2003, Ph.D. Thesis, A Multiwavelength Study of Normal and Starburst Galaxies in the Local Universe, Université de Paris, VII

Cherchneff, I. 1995, A\&SS, 224, 379

Coia, D., Metcalfe, L., McBreen, B., et al. 2005, A\&A, 430, 59

Coulais, A., \& Abergel, A. 2000, A\&AS, 141, 533

de Grijs, R., \& van der Kruit, P. C. 1996, A\&AS, 117, 19

Dopita, M. A., Groves, B. A., Sutherland, R. S., \& Kewley, L. J. 2003, ApJ, 583, 727

Dopita, M. A., Groves, B. A., Fischera, J., et al. 2005, ApJ, 619, 755

Draine, B. T., \& Li, A. 2007, ApJ, 657, 810

Draine, B. T., D. A. Dale, G. Bendo, et al. 2007, [arXiv: astro-ph/0703213]

Engelbracht, C. W., Kundurthy, P., Gordon, K. D., et al. 2006, ApJ, 642, L127

Fernandez-Soto, A., Lanzetta, K. M., Chen, H.-W., Pascarelle, S. M., \& Yahata, N. 2001, ApJS, 135, 41

Galliano, F. 2004, Ph.D. Thesis, Université de Paris, XI

Galliano, F. 2006, [arXiv: astro-ph/0610852]

Galliano, F., Madden, S. C., Tielens, A. G. G. M., et al. 2007, submitted

Geller, M. J., \& Huchra, J. P. 1983, ApJS, 52, 61

Genzel, R., \& Cesarsky, C. J. 2000, ARA\&A, 38, 761

Giuricin, G., Marinoni, C., Ceriani, L., \& Pisani, A. 2000, ApJ, 543, 178

Habart, E., Natta, A., \& Krügel, E. 2004, A\&A, 427, 179

Helou, G. 2004, ApJS, 154, 253

Hummel, E., Beck, R., \& Dettmar, R.-J. 1991, A\&AS, 87, 309

Irwin, J. A., \& Madden, S. C. 2006, A\&A, 445, 123

Kennicutt, R. C. Jr. 1998, ApJ, 498, 541

Kregel, M., \& van der Kruit, P. C. 2004, MNRAS, 352, 787

Kregel, M., \& van der Kruit, P. C. 2005, MNRAS, 358, 481

Kregel, M., van der Kruit, P. C., \& Freeman, K. C. 2004a, MNRAS, 351, 1247

Kregel, M., van der Kruit, P. C., \& de Blok, W. J. G., 2004b, MNRAS, 352, 768

Kregel, M., van der Kruit, P. C., \& Freeman, K. C. 2005, MNRAS, 358, 503

Laurent, O., Mirabel, I. F., Charmandaris, V., et al. 2000, A\&A, 359, 887

Le Page, V., Snow, T. P., \& Bierbaum, V. M. 2001, ApJS, 132, 233

Le Page, V., Snow, T. P., \& Bierbaum, V. M. 2003, ApJ, 584, 316

Lee, S.-W., Irwin, J. A., Dettmar, R.-J., et al. 2001, A\&A, 377, 759

Leitherer, C., Schaerer, D., Goldader, J. D., et al. 1999, ApJS, 123, 3

Lis, D. C., \& Leung, C. M. 1991, ApJ, 372, L107

Lu, N., Helou, G., Werner, M. W., et al. 2003, ApJ, 588, 199

Marri, S., \& White, S. D. M. 2003, MNRAS, 345, 561

Matsuura, M., et al. 2004, ApJ, 604, 791

Miller, S. T., \& Veilleux, S. 2003, ApJS, 148, 383 
Misiriotis, A., et al. 2001, A\&A, 372, 775

Pagani, L., Lagache, G., Bacmann, A., et al. 2003, A\&A, 406, L59

Peeters, E., Tielens, A. G. G. M., Boogert, A. C. A., Hayward, T. L., \& Allamandola, L. J. 2005, ApJ, 620, 774

Popescu, C. C., Misiriotis, A., Kylafis, N. D., Tuffs, R. J., \& Fischera, J. 2000, A\&A, 362, 138

Rapacioli, M., Joblin, C., \& Boissel, P. 2005, A\&A, 429, 193

Rhee, M.-H., \& van Albada, T. S. 1996, A\&AS, 115, 407

Sanders, D. B., \& Mirabel, F. 1996, ARA\&A, 34, 749

Sanders, D. B., Mazzarella, J. M., Kim, D.-C., Surace, J. A., \& Soifer, B. T. 2003, AJ, 126, 1607

Schwarzkopf, U., \& Dettmar, R.-J. 2001, A\&A, 373, 402

Sloan, G. C., Bregman, J. D., Geballe, T. R., Allamandola, L. J., \& Woodward, C. E. $1997, \mathrm{ApJ}, 474,735$
Smith, J. D. T., Draine, B. T., Dale, D. A., et al. 2007, ApJ, 656, 770 Starck, J.-L., Abergel, A., Aussel, H., et al. 1999, A\&AS, 134, 135

Tacconi-Garman, L. E., Sturm, E., Lehnert, M., Lutz, D., Davies, R. I., \& Moorwood, A. F. M. 2005, A\&A, 432, 91

Temi, P., Brighenti, F., \& Mathews, W. G. 2007a, ApJ, 660, 1215

Temi, P., Brighenti, F., \& Mathews, W. G. 2007b, [arXiv:astro-ph/0705.3710]

Tully, R. B. 1988, Nearby Galaxies Catalog (Cambridge: Cambridge Univ. Press) Turner, E. L., \& Gott III, J. R. 1976, ApJS, 32, 409

van Diedenhoven, B. 2004, ApJ, 611, 928

van Driel, W., Marcum, P., Gallagher, J. S. III et al. 2001, A\&A, 378, 370

Vijh, U. P., Witt, A. N., \& Gordon, K. D. 2004, ApJ, 606, L65

Visser, R., Geers, V. C., Dullemond, C. P., et al. 2007, A\&A, 466, 229

Vogler, A., Madden, S. C., Beck, R., et al. 2005, A\&A, 441, 491 